\title{
Goblet Cell Carcinoid of the Rectum in a Patient with Neurofibromatosis Type 1
}

\author{
Youngjin Kang · Jung-Woo Choi · Younghye Kim · Hwa Eun Oh · Ju-Han Lee · Young-Sik Kim \\ Department of Pathology, Korea University Ansan Hospital, Ansan, Korea
}

Patients with neurofibromatosis type 1 (NF-1) have rarely been reported to present with intraabdominal tumors including neuroendocrine tumors (NETs) and gastrointestinal stromal tumor (GIST), besides diagnostic features of café au lait macules, axillary or inguinal freckling, optic nerve gliomas, Lisch nodules, or neurofibromas. ${ }^{1}$ Specifically, the co-existence of periampullary NET and GIST has been suggested to be an indirect evidence for undisclosed NF-1. Herein, we report a NF-1 patient who presented with a combination of goblet cell carcinoid (GCC), NETs, and neurofibromatosis in the rectum, as well as a GIST in the jejunum.

\section{CASE REPORT}

A 46-year-old woman who congenitally had clinical features of NF-1 such as multiple neurofibromas of skin and café au lait macule has suffered from recurrent NETs in rectum since she received an operation for ovarian mucinous cystadenocarcinoma in 2010. The ovarian tumor showed neither recurrence nor distant metastasis during follow-up. She denied all the symptoms indicative of gastrointestinal diseases or carcinoid syndrome. Upon colonoscopy, the rectal mucosa revealed multifocal whitish patches, multiple biopsies from which revealed a coincidental occurrence of GCC, NETs, and neurofibroma. The abdominal computerized tomographic scan incidentally detected a submucosal mass in the jejunum. She underwent a low anterior resec-

Corresponding Author

Jung-Woo Choi, MD, PhD

Department of Pathology, Korea University Ansan Hospital, 123 Jeokgeum-ro, Danwon-gu, Ansan 15355, Korea

Tel: +82-31-412-6753, Fax: +82-31-412-5324, E-mail: nausika@korea.ac.kr

Received: January 27, 2016 Revised: February 24, 2016

Accepted: February 27, 2016 tion of rectum and a wedge resection of jejunum. During the operation, the appendix was unremarkable by inspection. Grossly, the involved segment of rectum was about $10 \mathrm{~cm}$ in length and contained four to five patchy indurated lesions measuring $5.2 \times$ $3.5 \mathrm{~cm}$ across in the largest one, which were limited to the mucosa and submucosa. Microscopically, the tumor consisted of small nests of signet-ring-like goblet cells (Fig. 1A, B), which positively stained with alcian blue $\mathrm{pH} 2.5$ (Fig. 1C). Immunohistochemical stains for CD56, synaptophysin (Fig. 1D), and chromogranin A revealed scattered neuroendocrine cells, leading to the diagnosis of rectal GCC. Adjacent to rectal GCC, there were multifocal minute grade 1 NETs according to the grading system of 2010 World Health Organization (WHO) classification of NETs (Fig. 2A, B). ${ }^{2}$ Neurofibromatosis in the background of GCC and NETs was confirmed by S-100 protein immunostain (Fig. 2C, D). The submucosal tumor in the jejunum was composed of bland-looking spindle cells which expressed both c-kit and CD34 by immunohistochemistry, and diagnosed as very low risk GIST (size, $1.0 \mathrm{~cm}$; mitotic count, < 1/50 high power fields) (Fig. 3A, B). Ten months after the operation, the patient was alive without recurrence or metastasis.

\section{DISCUSSION}

Even though GCC has been regarded as a mucin-producing NETs exclusively arising in the appendix, ${ }^{3}$ it is unsettled whether GCC should be classified as part of NETs or as variants of adenocarcinoma. The different histogenesis, the relative paucity of neuroendocrine cells, and more aggressive behavior have made GCC look different from NETs. It has been supposed that the dual neuroendocrine and intestinal differentiation in GCC emerge from undifferentiated stem cells at the base of the intestinal crypts, 

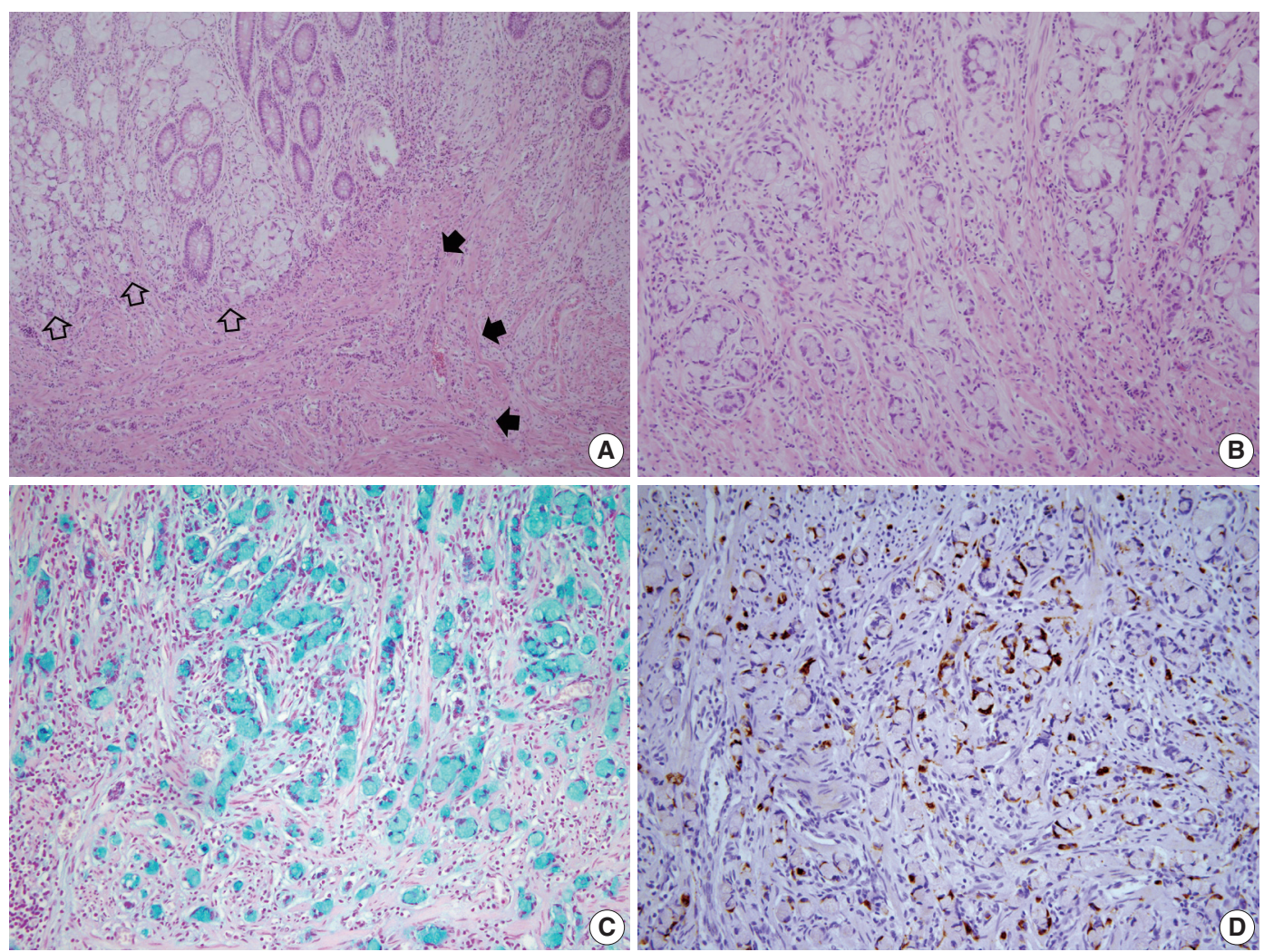

Fig. 1. The rectum shows a combined occurrence of goblet cell carcinoid (GCC, empty arrows) and neuroendocrine tumors (filled arrows) as well as neurofibromatosis (A). GCC is mostly composed of small nests of signet-ring-like goblet cells (B), and the cytoplasmic mucin is readily identified with alcian blue pH 2.5 (C). (D) Synaptophysin immunostain clearly shows a few scattered neuroendocrine cells in GCC.

whereas NETs initiate from subepithelial neuroendocrine cells in the mucosa. ${ }^{4}$ Moreover, the neuroendocrine cells of GCC are considerably fewer than those of NETs. As GCC has been more aggressive than NETs, adjuvant therapy after resection could be attempted commonly. Nevertheless, the genetic resemblance of GCC to NETs has precluded its being categorized as a subtype of adenocarcinoma. Indeed, GCC had allelic losses of 11q, 16q, or $18 \mathrm{q}$ which are frequent in gastrointestinal NETs, whereas KRAS, DPC4, or CTNNB1 mutations signaling for colorectal adenocarcinoma were undiscovered. ${ }^{5}$ The co-occurrence of GCC and NETs in this case might also implicate the close oncogenic relationship between them.

The rectum is an unusual site for GCC, and less than 10 cases of GCC arising in the rectum have been reported. ${ }^{6}$ Among them, there have been no patients with NF-1 as the present case, while a NF-1 patient with appendiceal GCC was recently described. ${ }^{7}$ Somatic inactivation of the NF1 has been the molecular hallmark of NF-1. A recent analysis has demonstrated that unlike sporadic GISTs, NF-1-related GISTs did not have activating mutations of KIT or PDGFRA, but showed somatic inactivation of NF1 more frequently. ${ }^{8}$ In addition, the tumor cells of gastric
NET in a NF-1 patient showed germline nonsense mutation and loss of heterozygosity of NF1. ${ }^{9}$ Therefore, the occurrence of GCC along with NET, GIST and neurofibromatosis in this patient suggest that GCC might be one of NF-1-related tumors, and might have arisen from NF1 gene dysregulation. However, the genetic association between GCC and NF-1 needs to be established further.

Rectal GCC should be differentiated from metastasis of appendiceal GCC and from signet ring cell carcinoma, which usually forms luminal space and is negative for neuroendocrine markers by immunohistochemistry. Although the present patient had ovarian mucinous cystadenocarcinoma, the microscopic features between the tumors of rectum and ovary were obviously different.

In conclusion, for the first time we report rectal GCC arising in a NF-1 patient. This case will contribute to broadening the scope of gastrointestinal tumors, which NF-1 patients can harbor. More data need to be accumulated to determine the biologic characteristics and the optimal treatment strategies for this rare tumor. 

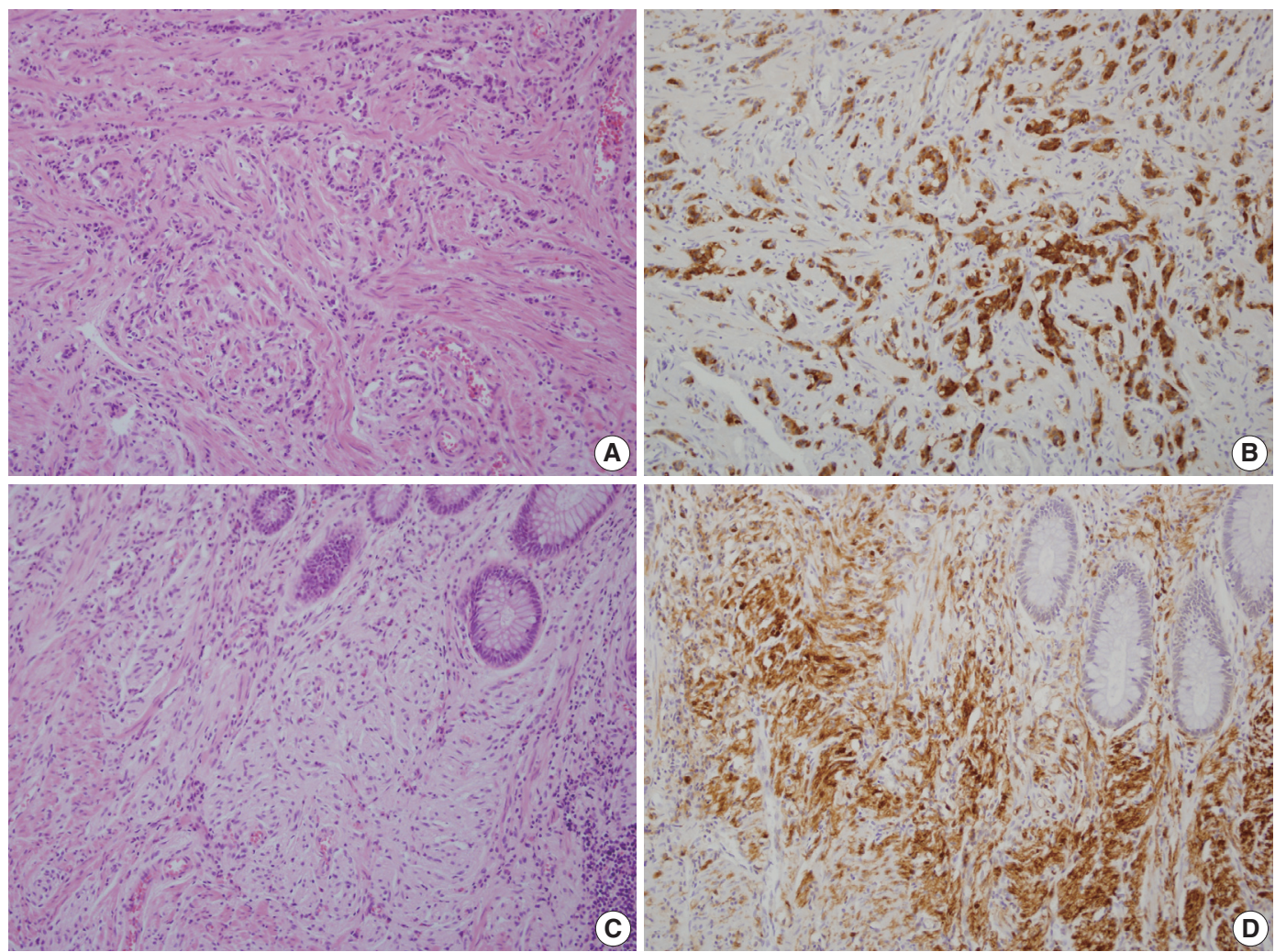

Fig. 2. Neuroendocrine tumors (NETs) consist of small uniform cells which exclusively form trabecular structures (A) and diffusely stained with synaptophysin (B). In the vicinity of both goblet cell carcinoid and NETs, proliferation of slender spindle cells morphologically compatible with neurofibromatosis (C) is evident with S-100 protein immunostain (D).
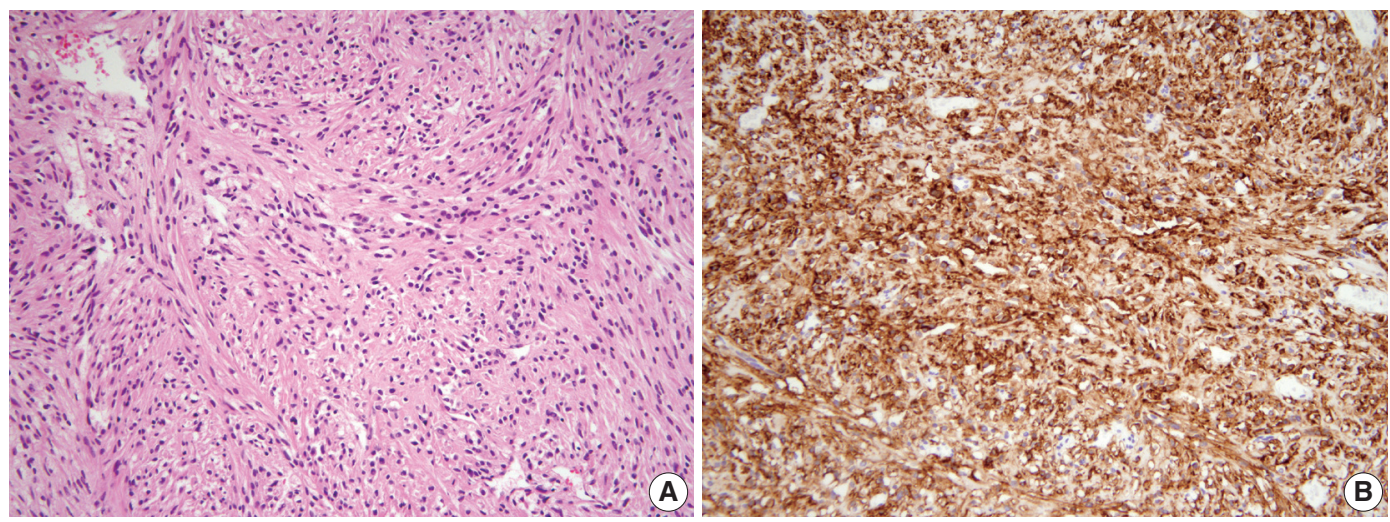

Fig. 3. The submucosal tumor in the jejunum is composed of bland-looking spindle cells (A) which highly expressed c-Kit by immunohistochemistry (B).

\section{Conflicts of Interest}

No potential conflict of interest relevant to this article was reported.

\section{Acknowledgments}

This work was supported by Basic Science Research Program through the National Research Foundation of Korea (NRF) funded by the Ministry of Science, ICT and Future Planning (grant number: 2013 R1A1A1058146).

\section{REFERENCES}

1. Agaimy A, Vassos N, Croner RS. Gastrointestinal manifestations of neurofibromatosis type 1 (Recklinghausen's disease): clinicopathological spectrum with pathogenetic considerations. Int J Clin Exp 
Pathol 2012; 5: 852-62.

2. Rindi G, Klimstra DS, Arnold R, et al. Nomenclature and classification of neuroendocrine neoplasms of the digestive system. In: Bosman FT, Carneiro F, Hruban RH, Theise ND, eds. WHO classification of tumours of the digestive system. 4th ed. Lyon: IARC Press, 2010; $13-4$.

3. Gui X, Qin L, Gao ZH, Falck V, Harpaz N. Goblet cell carcinoids at extraappendiceal locations of gastrointestinal tract: an underrecognized diagnostic pitfall. J Surg Oncol 2011; 103: 790-5.

4. Hofler H, Kloppel G, Heitz PU. Combined production of mucus, amines and peptides by goblet-cell carcinoids of the appendix and ileum. Pathol Res Pract 1984; 178: 555-61.

5. Stancu M, Wu TT, Wallace C, Houlihan PS, Hamilton SR, Rashid A. Genetic alterations in goblet cell carcinoids of the vermiform ap- pendix and comparison with gastrointestinal carcinoid tumors. Mod Pathol 2003; 16: 1189-98.

6. Yamabuki T, Omi M, Yonemori A, et al. Goblet cell carcinoid of the rectum with lymph node metastasis: report of a case. Surg Today 2011; 41: 1284-9.

7. Gregersen T, Holt N, Gronbaek H, Vogel I, Jørgensen LJ, Krogh K. Goblet cell carcinoid in a patient with neurofibromatosis type 1: a rare combination. Case Rep Gastrointest Med 2012; 2012: 185730.

8. Maertens O, Prenen H, Debiec-Rychter M, et al. Molecular pathogenesis of multiple gastrointestinal stromal tumors in NF1 patients. Hum Mol Genet 2006; 15: 1015-23.

9. Stewart W, Traynor JP, Cooke A, et al. Gastric carcinoid: germline and somatic mutation of the neurofibromatosis type 1 gene. Fam Cancer 2007; 6: 147-52. 